\title{
Parameterized Transmittance Model for Atmospheric and Surface Solar Radiations
}

\author{
J. Djampou Tchouankap¹, L. Akana Nguimdo ${ }^{2 *}$ \\ ${ }^{1}$ Environmental Energy Technologies Laboratory (EETL), Faculty of Sciences, University of Yaoundé, Yaoundé, Cameroon \\ ${ }^{2}$ Department of Electrical and Electronic Engineering, Faculty of Engineering and Technology, University of Buéa, \\ Buéa, Cameroon \\ Email: *languimdo@yahoo.fr
}

How to cite this paper: Tchouankap, J.D. and Nguimdo, L.A. (2020) Parameterized Transmittance Model for Atmospheric and Surface Solar Radiations. Atmospheric and Climate Sciences, 10, 81-99.

https://doi.org/10.4236/acs.2020.101004

Received: December 2, 2019

Accepted: January 17, 2020

Published: January 20, 2020

Copyright $\odot 2020$ by author(s) and Scientific Research Publishing Inc. This work is licensed under the Creative Commons Attribution International License (CC BY 4.0).

http://creativecommons.org/licenses/by/4.0/

\begin{abstract}
This work describes a transmittance model that evaluates global solar irradiation through the atmospheric column and at surface. The model is based on appropriate determination of the transmission coefficients of the different atmospheric constituents in a plane parallel layers frame and estimates the downward solar fluxes from the upper limit of the atmosphere. In testing this model, we first considered the purely molecular atmosphere to parameterize descending solar fluxes, which allowed us to estimate the attenuation due to atmospheric gases at specific times of the day when the irradiation at ground level is known. The results thus obtained show that the molecular atmosphere has a maximum reduction rate of incident flux (at the Top Of Atmosphere) of $20 \%$ with daily profiles that are homogeneous with extraterrestrial fluxes. Considering the turbid and cloudy atmosphere in which the multiple scattering phenomenon is taken into account, we obtain at ground level fluctuating profiles with attenuation rates reaching $64 \%$ depending on the time instant in the day. The comparison of our results with the experimental data obtained at the Yaoundé site on one hand and with the results of the CLIRAD-SW model on the other hand shows at monthly scale high correlation, of the order of 0.998 . Moreover at monthly time scale, the precision which for some hourly values is relatively low tends towards a net improvement on the seasonal scale where it extends over a narrow domain ranging from $0.02 \%$ to $1.66 \%$.
\end{abstract}

\section{Keywords}

Scattering, Absorption, Transmittance, Extraterrestrial Fluxes, Solar Irradiation 


\section{Introduction}

Thanks to the radiative impact of the atmospheric constituents, the solar radiation is attenuated as it travels through the terrestrial atmosphere. This attenuation is due to absorption by gases, scattering and absorption by solid (aerosols) and liquid (clouds) particles. During the last decades, numerous studies aiming at better understanding the nature of the atmospheric constituents, their origin as well as their radiative impact for appropriate consideration in the determination of energy transfer through the atmosphere were carried out [1]. Indeed, at the time where policy makers around the world are seeking to know with precision the impact of human action on the changes in terrestrial climate, significant disparities persist in radiative models results on the assessment of the global radiative budget. This disagreement is generally related to the parameterization of the radiative properties of clouds and aerosols in these models [1] [2]. However, knowledge of solar fluxes through the atmosphere not only makes possible the sizing of solar systems for energy production but also the monitoring of changes in the local heating/cooling profile of the atmosphere in order to predict future changes. Considering the case of aerosols in radiation estimation, Charlson et al. [3] showed that the modification of the reflection properties of sulfated aerosols in short wavelengths strongly influences planetary albedo. In this same paper, they also indicate that the effects of aerosols should be taken into account when assessing anthropogenic influences on past, present and future climate, as well as in the development of policies for the control of greenhouse gas emissions. Ramanathan et al. [4] showed that the effects of aerosols can lead to a weaker hydrological cycle, therefore influencing the availability and quality of fresh water, which is one of the major environmental problems of the $21^{\text {st }}$ century. Other researchers have focused on the determination of microphysical and optical properties through in situ measurements in order to obtain a more precise evaluation of these properties [5]. However, despite these notable advances in climate modeling and prediction, researchers remain limited, in particular by the financial means that reduce them to local measurements, which creates disagreement as to the data used in different models. The solution to this problem would reside in remote sensing that provides wider coverage of the atmosphere. This is the case of satellite data collected from the visible channel of the Meteosat7 satellite [6] [7] [8] [9]. These allow to better understand the functioning of the atmospheric system as a whole and to have access to the composition of the atmosphere from distance. In this same vein, mention can be made of the works of Aissani et al. [10], Chen Min et al. [11], which focus on methods for estimating total, direct and diffuse solar radiations at global scale, using atmospheric data from Moderate Resolution Imaging Spectroradiometer (MODIS) and the land parameters over the global area of the terrestrial ecosystem under aerial conditions.

Nevertheless, these methods and models may still exhibit some shortcomings in the sense that satellites have a limited time coverage and their estimations of 
surface shortwave irradiation does not agree with ground-based measurements in all localities [12]. The best compromise between these methods and models would ultimately lie in the quality of the absorption and scattering coefficients that are taken into account and that ultimately control their precision.

In this work, we propose to follow the downward solar radiation in different atmospheric layers located at various pressure levels from the Top of Atmosphere (TOA) to the surface, in order to determine the solar fluxes at altitude and surface in a plane parallel tropical atmosphere model that takes into account atmospheric gases, clouds and aerosols. The model resolves the diurnal cycle and cloud and aerosol fields are updated hourly [13] while gases follow Air Force Geophysical Laboratory (AFGL) [14] tropical standard profile. Flux calculation involves two-stream method and vertical integration of radiative fluxes through the atmosphere in an energy conservation scheme. Moreover, since the absorption and scattering coefficients not only are spectral but also dependent on the thermophysical conditions of the medium, we split the spectrum into 12 bands for the analysis of gases, clouds and aerosols, this allow us to take into account the spectral integral (flux adding method) in flux calculation. Section 2 of this paper deals with the absorption of solar radiation by atmospheric gases and describes the method for determining absorption coefficients. It also presents the scattering by gases as well as the modulation of fluxes by aerosols and clouds. This section ends with the presentation of our layered atmospheric model for the calculation of transmitivities and fluxes. Section 3 presents simulated fluxes obtained at ground level for a molecular atmosphere, then those obtained for turbid atmosphere and cloudy atmosphere separately and compares them with ground-based measurements on one hand and CLIRAD-SW model simulations on the other hand. The summary and conclusions are presented in Section 4.

\section{Methodology}

\subsection{Absorption by Gases}

The atmosphere consists of several radiatively active gases $\left(\mathrm{N}_{2}, \mathrm{O}_{2}, \mathrm{O}_{3}, \mathrm{H}_{2} \mathrm{O}\right.$, $\left.\mathrm{CO}_{2} \ldots\right)$ whose radiative properties are based typically on the determination of the absorption coefficients. These coefficients in turn depend on the intensity of the spectral lines and their broadening profiles. The main factors affecting the line spectrum are pressure, temperature, and molecular density, molar fraction of an active species and that of other species that act as upsettings in intermolecular collisions. Combining Boltzmann's law on energy levels distribution, Planck's law on monochromatic radiation and the Einstein's theory of molecular absorption, we obtain the expression

$$
S_{\eta}=\frac{8 \pi^{3} \eta}{3 h} \frac{g_{l} P}{k T Q(T)} P_{0}^{2}\left(1-\mathrm{e}^{-\frac{h C_{0} \eta}{k T}}\right) \mathrm{e}^{-\frac{E_{l}}{k T}}
$$

where $S_{\eta}$ is the intensity of the spectral line, $\eta$ the wave number $\left(\mathrm{cm}^{-1}\right), h$ the Planck constant, $k$ the Boltzmann constant, $T$ the temperature, $P$ the pressure. $Q(T)$ is the partition function of the molecule, $P_{0}$ the dipolar moment of the 
molecule, $g_{l}$ the degeneracy of the level $l, C_{0}$ the celerity of the light in the vacuum and $E_{l}$ the energy of the level $l$.

To determine the intensity of spectral lines in a ro-vibrational band, we consider the approximations of harmonic oscillator and rigid rotator, with additional assumptions that the bandwidth is small compared to the wave number at the center of the band and that only the $\mathrm{P}$ and $\mathrm{R}$ branches are important [15] [16]. For such a case Equation (1) can be adjusted as

$$
S_{\eta_{P}}=\frac{8 \pi^{3} \eta_{P}}{3 h} \frac{g_{l} P}{k T Q(T)} P_{0}^{2}\left(1-\mathrm{e}^{-\frac{h C_{0} \eta_{P}}{k T}}\right) \mathrm{e}^{-\frac{E_{l}}{k T}}
$$

With $\eta_{P}=\eta_{0}-\left(B_{v+1}+B_{v}\right) j+\left(B_{v+1}-B_{v}\right) j^{2}, j=1,2,3, \cdots$

$$
S_{\eta_{R}}=\frac{8 \pi^{3} \eta_{R}}{3 h} \frac{g_{l} P}{k T Q(T)} P_{0}^{2}\left(1-\mathrm{e}^{-\frac{h C_{0} \eta_{R}}{k T}}\right) \mathrm{e}^{-\frac{E_{l}}{k T}}
$$

With

$$
\eta_{R}=\eta_{0}+2 B_{v+1}+\left(3 B_{v+1}+B_{v}\right) j+\left(B_{v+1}-B_{v}\right) j^{2}, j=1,2,3,
$$

where $B_{v}$ is the moment of inertia of the molecule that depends on the vibrational energy level.

The profiles described above for the $\mathrm{P}$ and $\mathrm{R}$ bands consider that the spectral line is constant and centered on $\eta$. This does not reflect the reality because due to thermophysical conditions of the medium the line undergoes broadening phenomenon whose profiles have been described by Lorentz and Doppler among others. The broadening profile that we consider here is Voigt's profile because it tends to narrower profiles at low pressures and to wider profiles at high pressures in harmony with Lorentz and Doppler. The empirical Voigt profile, often used for trace gas detection [17] is written as follows:

$$
\phi_{V}\left(\eta-\eta_{0}\right)=\phi_{V}\left(\eta_{0}\right)\left((1-x) \exp \left(-0.693 y^{2}\right)+\frac{x}{1+y^{2}}+\beta\right)
$$

where

$$
\beta=0.016(1-x) x\left(\exp \left(-0.0841 y^{2.25}\right)-\frac{1}{1+0.0210 y^{2.25}}\right)
$$

with $x=\frac{\gamma_{L}}{\gamma_{V}}$ and $y=\frac{\left|\eta-\eta_{0}\right|}{\gamma_{V}}$.

$\gamma_{L}$ and $\gamma_{D}$ are respectively the half-widths of Lorentz and Doppler profiles. $\gamma_{L}$ increases with pressure and decreases with temperature while $\gamma_{D}$ increases with temperature.

$$
\phi_{V}\left(\eta_{0}\right)=\frac{1}{2 \gamma_{V}\left(1.065+0.447 x+0.058 x^{2}\right)}
$$

The half-width $\gamma_{V}$ of the Voigt profile is given by

$$
\gamma_{V}=0.5346 \gamma_{L}+\left(0.2166 \gamma_{L}^{2}+\gamma_{D}^{2}\right)^{1 / 2}
$$


Following this expression, the Voigt profile at high-pressure tends to Lorentz profile, when $x$ tends to 1 and to Gaussian profile at low-pressure, when $x$ tends to 0 .

Typical profile half widths for atmospheric species at various pressure levels follow Voigt's profile which is in the range $5 \times 10^{-3}$ to $2 \times 10^{-2} \mathrm{~cm}^{-1}$, quite less than the distance between rotational transitions of many molecules of atmospheric interest. Thus the use of Voigt broadening makes it possible to increase the sensitivity and the selectivity of the line to be absorbed. It then becomes possible to make measurements in the spectral regions where $\mathrm{H}_{2} \mathrm{O}$ and $\mathrm{CO}_{2}$ have strong and weak absorptions. The absorption coefficient of a molecule can then be written as

$$
k_{\eta}=S_{\eta} \cdot \phi_{V}\left(\eta-\eta_{0}\right)
$$

where $\phi_{V}\left(\eta-\eta_{0}\right)$ is the Voigt broadening profile.

It follows that the transmittance of a gas layer can be expressed as

$$
\tau=\exp \left(-k_{\eta} N \frac{\Delta P}{\rho g}\right)
$$

where $N$ is the number of gas molecules in the layer, $\Delta P$ (mbar) the pressure variation across the layer, $\rho\left(\mathrm{kg} \cdot \mathrm{m}^{-3}\right)$ the density of the gas, $g(\mathrm{~N} / \mathrm{kg})$ the specific gravity and $k_{\eta}\left(\mathrm{cm}^{-1}\right)$ the average Absorption coefficient of the molecules.

\subsection{Gas Scattering}

Mc Cartney [18] has shown that the total diffusion cross section per molecule of gas is described by

$$
\sigma_{R \lambda}=\frac{24 \pi^{3}}{N_{0}^{2} \lambda^{-4}}\left(\frac{n_{0}^{2}-1}{n_{0}^{2}+2}\right)^{2}\left(\frac{6+3 \xi}{6-7 \xi}\right)
$$

where $\xi$ is the depolarization factor, $N_{0}$ the density of molecules $(2.547305 \times$ $10^{25} \mathrm{~m}^{-3}$ at $15^{\circ} \mathrm{C}$ ), $n_{0}$ the integer part of the refractive index of air and $\lambda$ the wavelength. The cross section $\sigma_{R \lambda}$ is typically given in units of square centimeters.

For a given volume of gas, the total Rayleigh scattering coefficient $\varphi$ is given by the product of the Rayleigh cross section per molecule and the molecular density $N$ at a given level of altitude $Z$, pressure $P$ and temperature $T$ [19].

$$
\varphi(z, \lambda)=N(z) \sigma_{R \lambda}(\lambda)
$$

The Rayleigh optical thickness at altitude $Z_{0}$ is then given as the integral of the total volume scattering coefficient $\varphi$ from $Z_{0}$ to the TOA, according to the relationship

$$
\tau_{R \lambda}(\lambda)=\int_{Z_{0}}^{\infty} \varphi(z, \lambda) \mathrm{d} z
$$

This equation was reevaluated using the most recent determinations of $\xi=$ 0.0279 [20] and $n_{0}$ (Peck and Reeder, [21]). The calculations were thus processed by Gueymard [22] [23] with a resolution of $2 \mathrm{~nm}$ in the band ranging 
from 250 to $1000 \mathrm{~nm}$ and $5 \mathrm{~nm}$ beyond $1000 \mathrm{~nm}$. Adjustment of the curve by the least squares method was then used to develop the following equation for transmittance

$$
\tau_{R \lambda}=P /\left(a_{1} \lambda^{4}+a_{2} \lambda^{2}+a_{3}+a_{4} \lambda^{-2}\right)
$$

where $P$ is the relative pressure defined by $P=p / p_{0}$ with $p$ being the local pressure, $p_{0}$ the atmospheric pressure at surface, $a_{1}=117.2594 \mu \mathrm{m}^{-4}$, $a_{2}=-1.3215 \mu \mathrm{m}^{-2}, a_{3}=3.2073 \times 10^{-4}, a_{4}=-7.6842 \times 10^{-5} \mu \mathrm{m}^{2}$

Equation (13) reproduces the values obtained with Equation (12) with a difference of less than $0.01 \%$ over the entire spectrum [23].

\subsection{Microphysical and Optical Properties of Clouds and Aerosols}

The impact of clouds (greenhouse effect, albedo...) on the atmospheric radiative budget apart from their spatial and temporal coverage depends on their microphysical (Effective radius, Liquid and Ice Water Contents) and optical (optical thickness, albedo, asymmetry factor) properties [24]. Low atmosphere clouds for example, are essentially liquid. The attenuation by these is due either to absorption, or scattering of the radiation in a new direction and is characterized by the extinction coefficient $k_{e x}(\lambda, m)$ given along a path $\mathrm{dz}$ by

$$
k_{e x}(\lambda, m)=\int_{0}^{\infty} \pi r^{2} S_{e x}(r, \lambda, m) n(r) \mathrm{d} r
$$

where $S_{e x}$ denotes the extinction efficiency which quantifies the attenuation of the incident radiation by a particle, $\lambda$ the wavelength, $m$ the complex refractive index, $n(r)$ the particle density and $r$ the radius of the particle. This extinction is related to the liquid water content (LWC) by the relationship

$$
\frac{\mathrm{LWC}}{k_{e x}}=\frac{\frac{4}{3} \rho_{w} \int_{0}^{\infty} \pi r^{3} n(r) \mathrm{d} r}{\int_{0}^{\infty} \pi r^{2} S_{e x}(r, \lambda, m) n(r) \mathrm{d} r}
$$

In the case of a cloud consisting of large water droplets (spherical shape), $S_{e x}$ tends to 2 in the visible domain [25]. In this case Equation (15) reduces to

$$
\frac{\mathrm{LWC}}{k_{e x}}=\frac{\frac{4}{3} \rho_{w} \int_{0}^{\infty} \pi r^{3} n(r) \mathrm{d} r}{2 \int_{0}^{\infty} \pi r^{2} n(r) \mathrm{d} r}
$$

But the effective radius of a cloud particle is defined as $r_{e f f}=\frac{\int_{0}^{\infty} r^{3} n(r) \mathrm{d} r}{\int_{0}^{\infty} r^{2} n(r) \mathrm{d} r}$ then,

$$
k_{e x}=\frac{3 \mathrm{LWC}}{2 \rho_{w} r_{e f f}}
$$

It follows that the extinction coefficient is proportional to the ratio of the water content to the effective radius of the particle. This coefficient is then integrated over the thickness of the atmospheric column to deduce the optical thickness $\tau$ which quantifies the attenuation of the radiation along a path in the cloud. 
It reflects the ability of the medium to absorb and scatter this radiation.

$$
\tau=\int_{0}^{h} \sigma_{e x}(\lambda, m, Z) \mathrm{d} Z
$$

This work integrates three types of clouds as described by Akana and Njomo [13] for the Yaoundé station. The low cloud that ranges from $1 \mathrm{~km}$ to $3 \mathrm{~km}$ is in liquid state. The mid-cloud is a mixed-phase cloud (liquid-solid) and is found in the range $4 \mathrm{~km}$ to $8 \mathrm{~km}$ height and finally the high cloud that extends from 10 $\mathrm{km}$ to $18 \mathrm{~km}$ is entirely in solid state. The model also integrates two layers of aerosols which are the Planetary Boundary Layer (PBL) that extends from the surface to $1 \mathrm{~km}$ height and the Upper Layer (UL) that extends from $1 \mathrm{~km}$ to 5 $\mathrm{km}$.

\subsection{Multiple Scattering and Transmitivity}

To produce a realistic parameterization of the irradiation, a certain number of approximations are made as regards the vertical variability of the density of atmospheric particles and the spectral variability of their optical properties. In this study, the heterogeneous atmosphere is discretized in 50 plane and parallel layers at different pressure levels. This structure corresponds to the AFGL profile [14] for a standard tropical atmosphere. Following Chou and Suarez [26], Terrasova and Cavalcanti [27], the solar spectrum is divided into 12 spectral bands of which 8 in the ultraviolet, one in the visible and 3 in the infrared. The three types of clouds identified as low, middle and high clouds are separated by altitudes of about $700 \mathrm{hPa}$ and $400 \mathrm{hPa}$. Similarly, we integrate two layers of aerosols so one between the surface and $904 \mathrm{mbar}$ and the other that culminates at 559 mbar. Unlike Chou and Suarez [26], all gases are assumed to be active across the spectrum since the absorption coefficients are not pre-calculated.

For each cloudy and turbid atmospheric layer and for each spectral band, the effective optical thickness, single scattering albedo and asymmetry factor are calculated. The transmissivity $T$ and the reflectivity $R$ of a layer illuminated by a direct beam are calculated from the $\delta$-Eddington approximation. This operation uses the discrete ordinate algorithm of Stamnes et al. [28] to determine the transmissivity and reflectivity of a layer at different angles of incidence. Diffuse transmissivity $\bar{T}$ and reflectivity $\bar{R}$ are then obtained by averaging the transmissivity and reflectivity of the direct beam over $2 \pi$ solid angle. King and Harshvardhan [29] showed that the error induced on quantities by this approximation is $5 \%$. Considering a layer divided into an upper sub-layer (a) and a lower sub-layer (b), direct incident radiation at the upper boundary of the atmospheric layer undergoes direct transmittance to penetrate the layer. The radiation emerging at the lower limit of the layer is due not only to the absorption inside the layer but also to the forward scattering. It is said that it undergoes a global transmittance (direct and diffuse). The remaining part can be reflected upward and then downward at the upper limit of the layer before emerging below. Although the wave fades gradually, it is accepted that this phenomenon of double reflection then transmission occurs indefinitely inside the homogeneous layer 
[30]. The average contribution of the layer is obtained at mid-altitude where the pressure level is $\frac{1}{2}\left[P_{i}+P_{i+1}\right]$ using two-stream method. By separating the direct and diffuse components of the radiation, the expression of the transmittance of the layer is given by [26]

$$
T_{a b}\left(\mu_{0}\right)=\mathrm{e}^{-\tau_{a} / \mu_{0}} T_{b}\left(\mu_{0}\right)+\bar{T}_{b}\left\{\mathrm{e}^{--\tau_{a}} \bar{\mu}_{0} R_{a} R_{b}\left(\mu_{0}\right)+\left[T_{a b}\left(\mu_{0}\right)-\mathrm{e}^{-\frac{\tau_{a}}{\mu_{0}}}\right]\right\} /\left(1-\bar{R}_{a} \bar{R}_{b}\right)
$$

where $T_{a b}\left(\mu_{0}\right), \mathrm{e}^{-\tau_{a} / \mu_{0}}$ and $T_{a b}\left(\mu_{0}\right)-\mathrm{e}^{-\frac{\tau_{a}}{\mu_{0}}}$ are the total, direct and diffuse transmittances respectively. $R_{a}$ and $R_{b}$ are direct reflectances of the sub-layers (a) and (b) respectively, $\bar{R}_{a}$ and $\bar{R}_{b}$ are the reflectances of these sub-layers respectively when they are illuminated by diffuse radiation.

\subsection{Calculation of Transmittances and Fluxes}

The solar flux that emerges from an atmospheric layer is expressed as $I_{L}=\tau I_{0}$ where $\tau$ is the product of the transmitivities of all species contained in the layer. For a layer containing $n$ species, we thus obtain $\tau=\prod_{i=1}^{n} \tau_{i}$ where $\tau_{i}$ is the transmittance of species $i$ in a given spectral band. The solar spectrum which extends from $0.175 \mu \mathrm{m}$ to about $10 \mu \mathrm{m}$ is divided into spectral bands according to the model described above to better integrate the spectral behavior of atmospheric molecules in the attenuation of solar fluxes. If $\Gamma$ is the total transmittance of the layer for all $n$ gases and in all $m$ spectral bands, then the overall flux transmitted is $I_{1}=\Gamma \cdot I_{0}$ with

$$
\Gamma=\sum_{j=1}^{m} r_{j}\left(\prod_{i=1}^{n} \tau_{i}\right)
$$

where $r_{j}$ is the fraction of extraterrestrial solar flux in the band $j$. The transmitted flux is therefore

$$
I_{1}=I_{0} \sum_{j=1}^{m} r_{j}\left(\prod_{i=1}^{n} \tau_{i}\right)
$$

This emerging flux is taken as incident to the adjacent layer. Thus, the solar flux emerging at the $k^{\text {th }}$ layer is

$$
I_{k}=I_{0} \cdot \prod_{k=1}^{n} \Gamma_{k}
$$

The downward flux is thus calculated step by step through the 50 layers under different atmospheric conditions and then compared to the ground-based data measured by Laboratoire de Recherche Energétique (LRE, Yaoundé) [31] [32].

\subsection{Data Description}

Measurements of total and diffuse solar radiation have been conducted in Cameroon using Eppley PSP Pyranometers distributed in ten meteorological stations across the country. The accuracy of these devices calibrated for hemispherical integration was $\pm 3 \%-4 \%$. The devices were producing incident solar flux with 10 seconds resolution. Data were then averaged over a period one hour 
and stored as the value of the total or diffuse flux for the corresponding hour [31] [32]. This process was repeated during the entire day from $6 \mathrm{AM}$ to $6 \mathrm{PM}$, giving 12 values for each flux on each day. In this study, we use solar radiation data for the Yaoundé station $\left(11^{\circ} 51^{\prime} \mathrm{E}, 3^{\circ} 86^{\prime} \mathrm{N}\right)$ which is the most complete data series.

\section{Results}

The daily variations of simulated and measured global solar fluxes are presented for each month of the experimentation period. The measured data are the monthly hourly averages of the global radiation [31] [32]. The corresponding simulations are done hour after hour as described in Akana and Njomo [30].

\subsection{Case of Molecular Atmosphere}

Figure 1 shows simulated solar fluxes at surface for a molecular atmosphere and compares them with extraterrestrial fluxes and ground-based solar radiation measurements for February, May, August, and October. There seems to be significant attenuation of irradiation early in the morning (6:30-8:00 am) and late afternoon (16:00-17:30 pm) where the results of simulations are comparable to measurements. The reduction of fluxes in these time periods reaches $60 \%$. The gas absorption and the Rayleigh scattering being theoretically the only credible modulation modes, this reduction is in fact due to the long optical path indicated by the zenith angle through which the radiation passes and which greatly reduces the incident flux. However, there is quite low attenuation in the middle
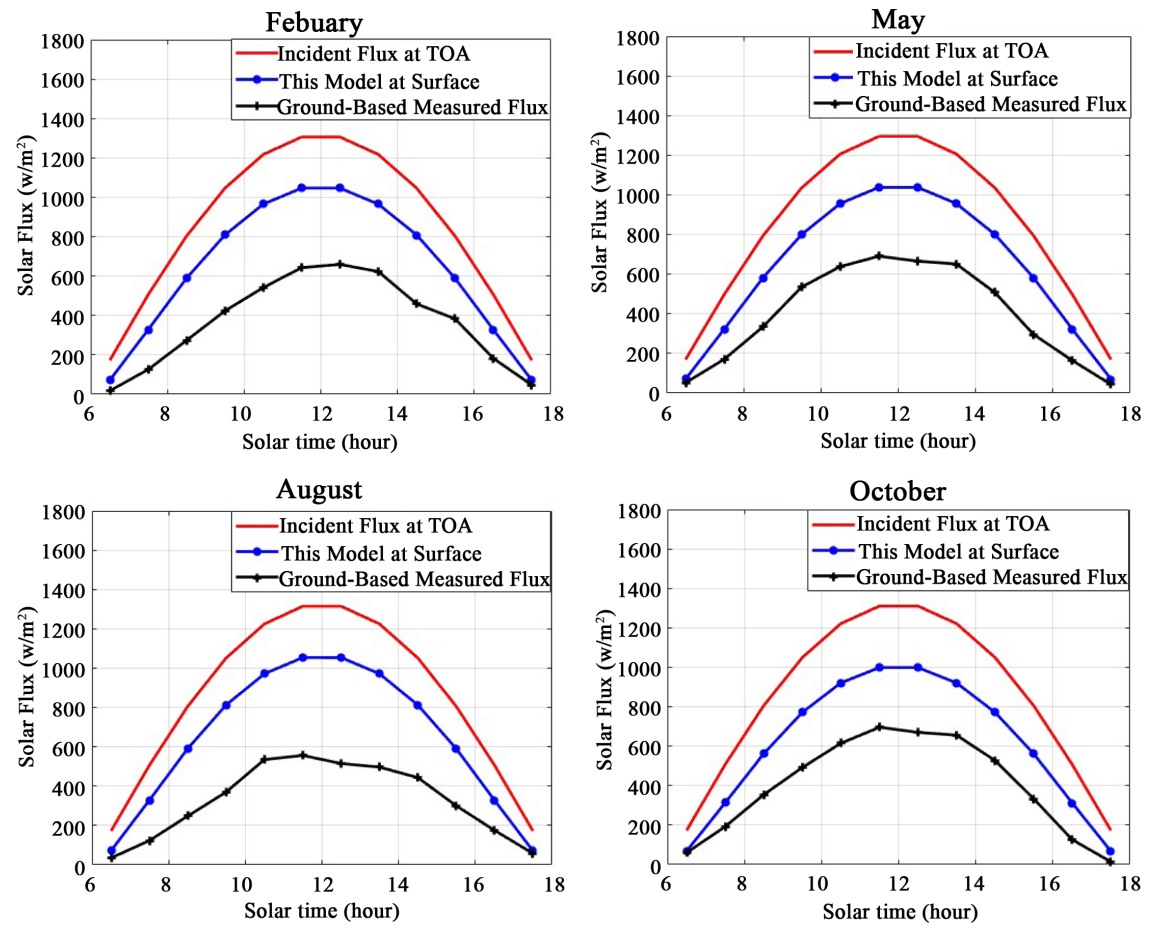

Figure 1. Daily evolution of simulated solar fluxes at surface for a molecular atmosphere and comparison with ground-based measurements as well as TOA fluxes. 
of the day, $20 \%$ at best. The very significant difference observed between simulations and measurements during these hours and over the four months reveals the importance of clouds and aerosols in the modulation of fluxes through the atmosphere. Indeed, in the absence of these, radiation modulation through the atmospheric column is globally poor and the fluxes received at surface are simply the expression of a limit or extreme situation which is very often used as standard in the prediction of solar fluxes. This atmospheric model is therefore not suitable for discussing the accuracy of radiative models.

\subsection{Cloudy and Turbid Atmosphere}

Following the limitation of molecular atmosphere as indicated previously, we first studied separately the impact of aerosols and clouds in the attenuation of solar irradiation for the same periods. Figure 2 shows the profile of global solar fluxes at surface for cloudy (green) and turbid (red) atmospheres added to the flux at TOA. It appears that the most important daily attenuations are observed early in the morning and latest in the evening with greater impact of clouds since the reduction of solar flux in cloudy sky reaches $70 \%$ (green curve) compare to a maximum of $60 \%$ for turbid sky (red curve).

There is a weaker reduction of around $36 \%$ in the middle of the day and for every month that is naturally due to the intense sunshine at that period. However, the maximum cloud reduction noted during April (rainy season) can be the result of high cloud density that quantitatively highlights optical thicknesses and backward scattering.
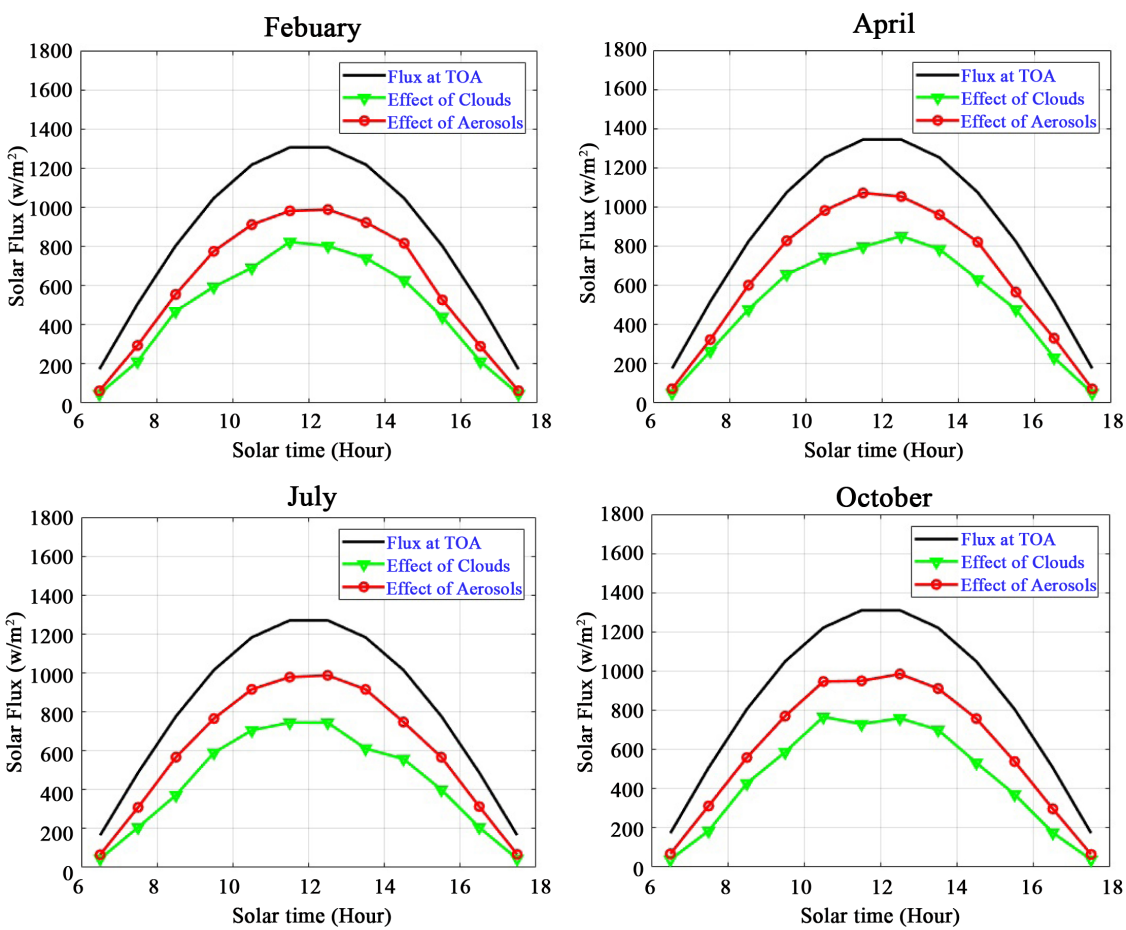

Figure 2. Impact of clouds (green curve) and aerosols (red curve) in the attenuation of solar fluxes. 
Our model is then used to infer solar fluxes in the case of a real atmosphere. The detailed calculations of the simulations extend over the spectral range [0.175 $\mu \mathrm{m}-10 \mu \mathrm{m}]$ and integrate all atmospheric components radiatively active in this spectrum (gas, clouds and aerosols). Figure 3 compares our model outputs with the CLIRAD-SW model fluxes at surface and with ground-based measurements over a one-year period from January to December at the Yaoundé site. The CLIRAD-SW model seems to reproduce almost perfectly measurements with relative errors of less than $1 \%$. This quasi-superposition is also characterized by correlation coefficients ranging from 0.99 to 1 , thus testifying to the fact that CLIRAD-SW is a credible reference for comparing our model. Indeed, our simulations with respect to the graphs of Figure 3 are superimposed on the measurements only on several time slots of the day and these slots vary from one month to another. However, with the exception of November (see Akana and Njomo, [13]), the correlation coefficients with measurements are significant $(>0.98)$ although lower than in the case with CLIRAD-SW model. This relative low precision of our model might be due to a partial consideration of the effects of water in the infrared since just 3 spectral bands are considered there despite a recognized very strong activity in this region of the spectrum. Moreover, the CLIRAD-SW whose results are presented here uses 75 spatial discrete layers, which could bring about a more sensitive refinement when calculating the extinction coefficients and thus the transmittances compared to our model. Nevertheless, our simulations show for the months of March and April correlations coefficients of respectively 0.9945 and 0.9944 , which is of good omen as to the accuracy of our model.

Furthermore, we illustrate in Figure 4 the linear regression curves between measured and simulated fluxes for the same periods. These graphs model the similarities between the dependent variable $Y$ (measured fluxes) and the predictive variable $X$ (simulated fluxes) and allow to further compare our simulation results with measurements. In doing this, we tested two distinct formulations including $Y=\beta_{0}+\beta_{1} X$ and $Y=\beta_{1} X$ where $\beta_{0}$ is the intercept, $\beta_{1}$ the slope (or regression coefficient). On observation, these two lines are all close to the first bisector, a sign of a relatively good agreement between the two sets of data. However, it should be noted that the adjustment by the function $Y=\beta_{0}+\beta_{1} X$ better reproduces the measurements on almost all the months of the year since it passes through the maximum number of data.

The main features of this regression analysis are summarized in Table 1. It can be seen that though the correlations coefficients are good enough to indicate how close the simulations are to measurements, an effort should be laid on the precision so as to reduce the relative gap between the two datasets.

\subsection{Seasonal Distribution of Ground Fluxes}

This paragraph, with regard to the indeed small differences between our simulations and the measurements on a monthly time scale, is concerned with seasonal distributions of fluxes at surface. In fact, seasonal, annual or interannual trends 

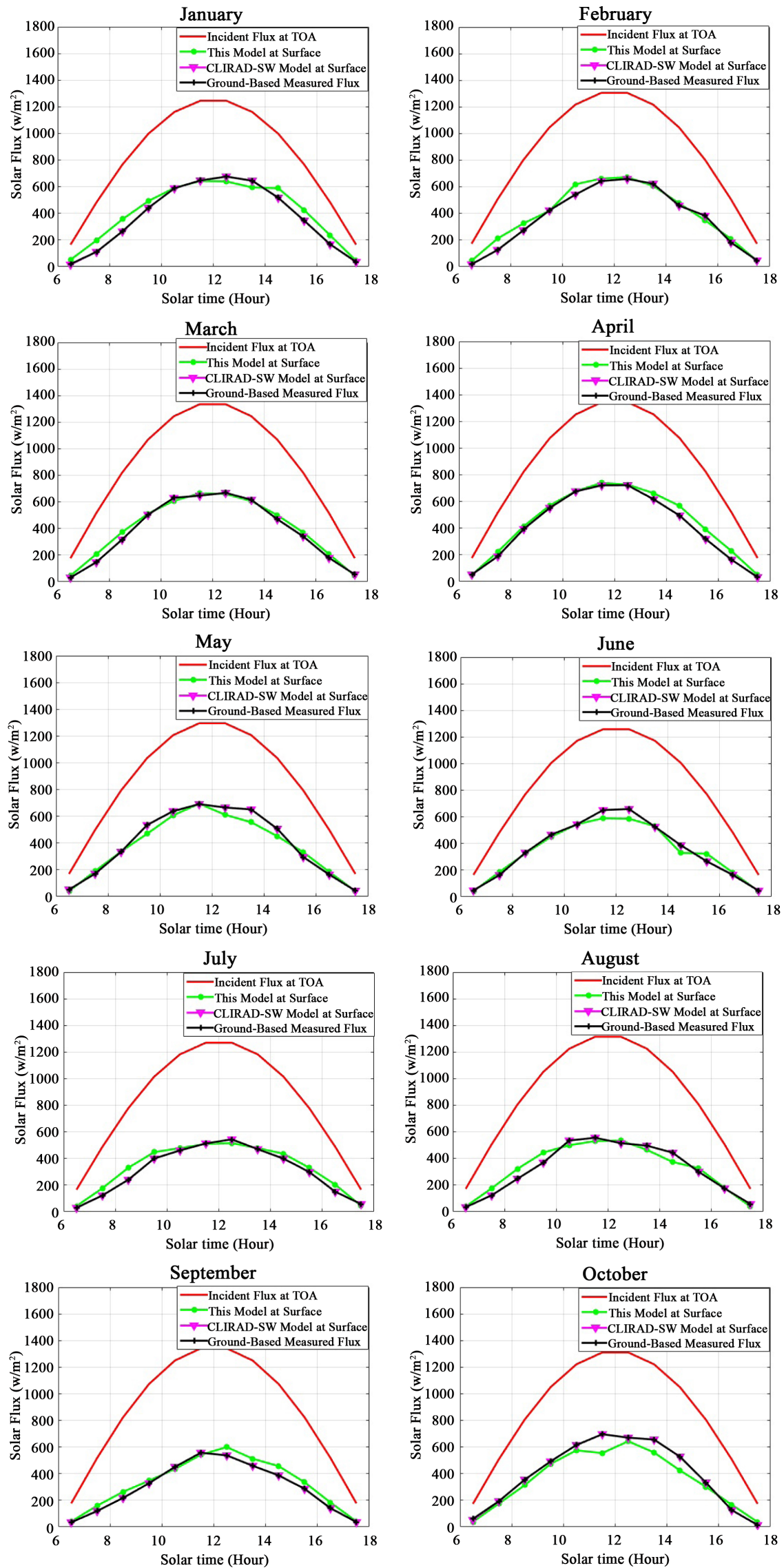

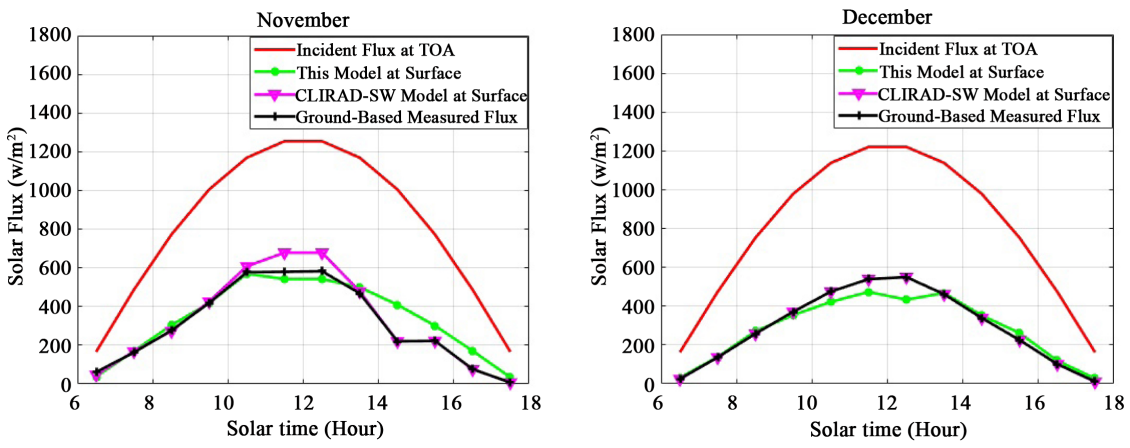

Figure 3. Daily profile of simulated solar fluxes at surface using this model for real atmosphere and comparison with ground-based measurements as well as TOA fluxes.

Table 1. Correlation and regression coefficients relating simulated and measured fluxes for each month.

\begin{tabular}{cccc}
\hline Month & Correlation Coefficient & Relative error en \% & Regression Coefficient \\
\hline January & 0.9837 & 1.63 & 0.8907 \\
February & 0.9882 & 1.18 & 0.9676 \\
March & 0.9945 & 0.55 & 0.9533 \\
April & 0.9945 & 0.55 & 0.9862 \\
May & 0.9899 & 1.01 & 0.8987 \\
June & 0.988 & 1.2 & 0.9012 \\
July & 0.9835 & 1.65 & 0.9447 \\
August & 0.9721 & 2.79 & 0.9169 \\
September & 0.9881 & 1.19 & 1.0095 \\
October & 0.9866 & 1.34 & 0.8401 \\
November & 0.9529 & 4.72 & 0.8604 \\
December & 0.9827 & 1.73 & 0.8348 \\
\hline
\end{tabular}

in climate study most often contain more realistic information than simple monthly variations. Note in this context that Yaoundé has four seasons including the little rainy season (March-June), the heavy rainy season (September-November), the little dry season (July-August) and the heavy dry season (December to February). Table 2 presents the correlation coefficients, the relative errors as well as the regression coefficients connecting the simulated and measured fluxes for the four seasons.

One can notice from the table that the relative error is more comfortable at the seasonal time scale with a maximum of $1.66 \%$, indicating a clear improvement over the monthly distribution. Similarly, correlation and regression coefficients increase significantly and this can be seen as a sign of a good accuracy of our simulations. This trend is confirmed by Figure 5 that shows the seasonal variations of hourly average fluxes for the entire experimental period as well as the linear regression curves between the results of this model and the measurements. We observe a better superposition of the two sets of data especially for the little rainy season (March-June). 

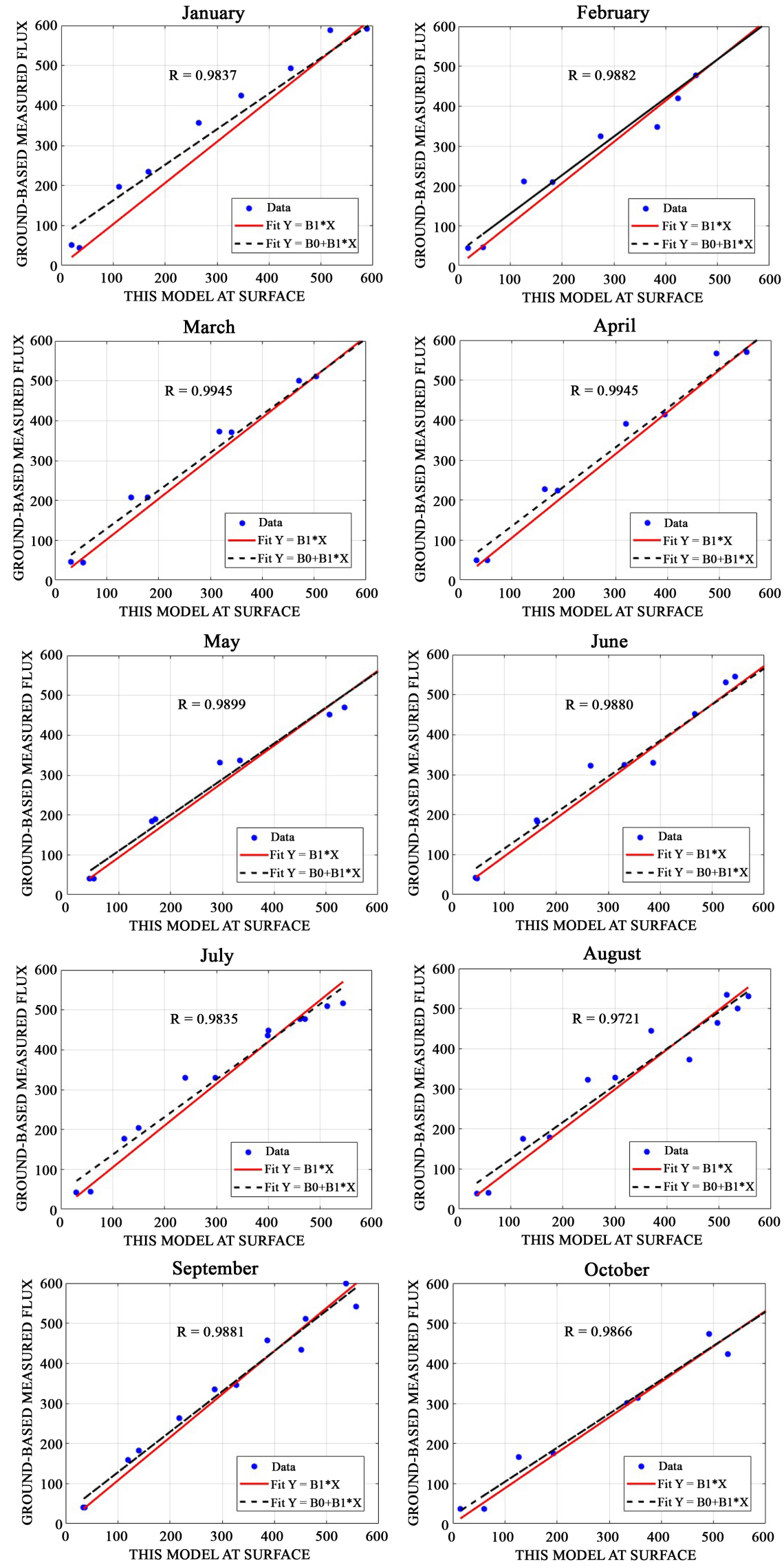

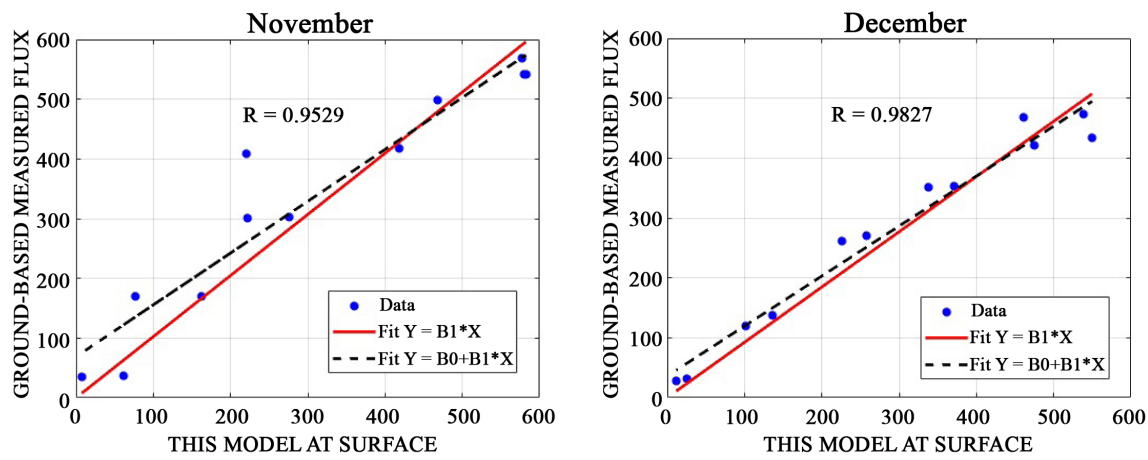

Figure 4. Linear regression curves for the various months of the experimental period.
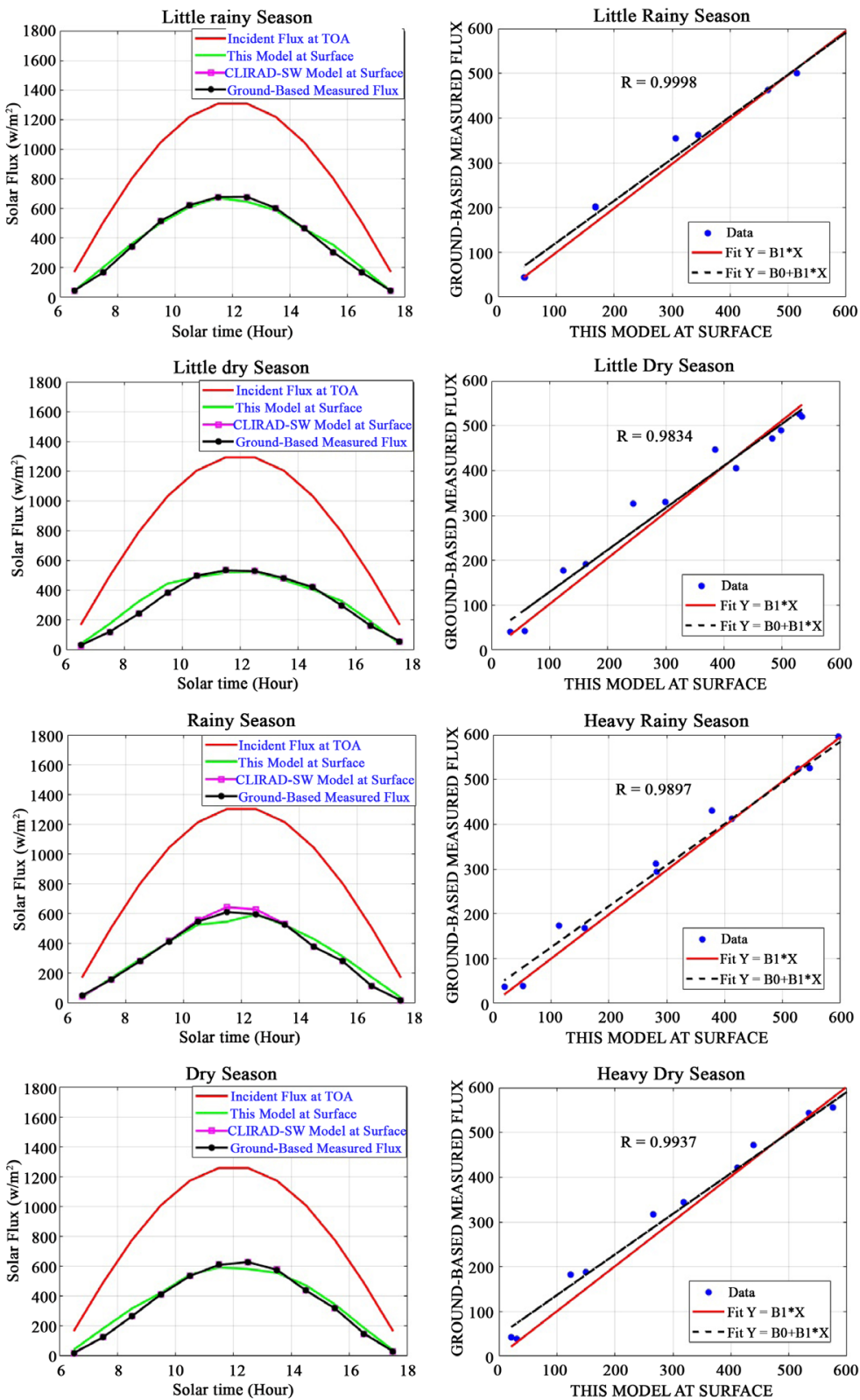

Figure 5. Comparison of seasonal tendencies of simulated and measured solar fluxes at surface and linear regression curves. 
Table 2. Correlation and regression coefficients relating simulated and measured fluxes for each season.

\begin{tabular}{cccc}
\hline Seasons & Correlation Coefficient & Relative error (\%) & Regression Coefficient \\
\hline Little rainy Season & 0.9998 & 0.02 & 0.9082 \\
Little dry Season & 0.9834 & 1.66 & 0.9369 \\
Heavy Rainy Season & 0.9897 & 1.03 & 0.9201 \\
Heavy Dry Season & 0.9937 & 0.63 & 0.9069 \\
\hline
\end{tabular}

However, it is noticeable that for the little dry season, although the correlation is good, the flux difference that existed at the monthly scale persists for the season. This would be due to the presence of a heavy cloud cover during the month of August that is generally observed on the site and that covers almost all the southern part of Cameroon due to the Intertropical Convergence Zone (ICZ). For the rest of seasons, a slight difference persists between the data in the time slot from 11:00 am to 13:00 pm. It should be noted that contrary to the monthly distributions, the time intervals where this discrepancy is noted become confined as we move to seasonal profiles. Finally, the monthly as well as the seasonal trends in harmony with the measurements and the computation time of our model which is shorter compared to CLIRAD-SW could viewed as key factors indicating that this model is suitable for the evaluation of the vertical profiles of solar irradiation through the atmosphere. However, further work is needed on spectral integration to refine the optical characterization of water vapor in the infrared region.

\section{Conclusion}

This study is a step towards the construction of a simple and flexible parameterized model for the evaluation of short wavelength fluxes through the atmosphere. It aims at regionally documenting the daily and seasonal variations of the solar fluxes necessary not only for an adequate dimensioning of the solar systems of energy generation but also for a more accurate characterization of the local variations of the atmospheric temperature, relevant factor of the equilibrium of the Earth's climate. Atmospheric gases are described by their spectral absorption coefficients whereas clouds and aerosols are by their optical thicknesses and simple scattering albedo. Unlike other models, the spectral properties of the gases are directly calculated in the code. This significantly reduces the calculation time. Since our atmosphere is discretized vertically in layers, the twoflux method allows us to determine the transmittance of each of them in order to evaluate fluxes. The results generated by this model for the city of Yaoundé correlate well with measurements made at ground level on a monthly time scale and more on a seasonal scale (correlation coefficients $>0.99$ ). Similarly, the relative differences between the simulation results and the measurements are on the average of $0.55 \%$ monthly and $0.02 \%$ seasonally. Despite these indicators of the quality of the model, it would be advisable to improve it especially with regard to 
the water vapor in the infrared in order to obtain a precision comparable to rigorous models.

\section{Conflicts of Interest}

The authors declare no conflicts of interest regarding the publication of this paper.

\section{References}

[1] Harshvardhan, Schwartz, S.E., Benkovitz, C.M. and Guo, G. (2002) Aerosol Influence on Cloud Microphysics Examined by Satellite. Journal of the Atmospheric Sciences, 59, 714-725. https://doi.org/10.1175/1520-0469(2002)059<0714:AIOCME >2.0.CO;2

[2] Fu, Q. (2007) A New Parameterization of an Asymmetry Factor of Cirrus Clouds for Climate Models. Journal of the Atmospheric Sciences, 64, 4144-4154.

[3] Charlson, R.J., Schwartz, S.E., Hales, J.M., Cess, R.D., Coakley Jr., J.A., Hansen, J.E. and Hofmann, D.J. (1992) Climate Forcing by Anthropogenic Aerosols. Journal of the Atmospheric Science, 255, 423-430. https://doi.org/10.1126/science.255.5043.423

[4] Ramanathan, V., Crutzen, P.J., Kiehl, J.T. and Rosenfeld, D. (2001) Aerosols, Climate, and the Hydrological Cycle. Journal of Geophysical Research, 106, 2837128398.

[5] Remer, L.A. and Kaufman, Y.J. (2006) Aerosol Direct Radiative Effect at the Top of the Atmosphere Overcloud Free Ocean Derived from Four Years of MODIS Data. Atmospheric Chemistry and Physics, 6, 237-253.

https://www.atmos-chemphys.org/acp/6/237/

https://doi.org/10.5194/acp-6-237-2006

[6] Moulin, C., Guillard, F., Dulac, F. and Lambert, C.E. (1997) Long-Term Daily Monitoring of Saharan Dust Load over Ocean Using Meteosat ISCCP-B2 Data, 1, Methodology and Preliminary Results for 1983-1994 in the Mediterranean. Journal of Geophysical Research: Atmospheres, 102, 16947-16958. https://doi.org/10.1029/96JD02620

[7] Legrand, M., Plana-Fattori, A. and N'doumé, C. (2001) Satellite Detection of Dust Using the IR Imagery of Meteosat: 1. Infrared Difference Dust Index. Journal of Geophysical Research, 106, 18251-18274. https://doi.org/10.1029/2000JD900749

[8] Chiapello, I. and Moulin, C. (2002) TOMS and METEOSAT Satellite Records of the Variability of Saharan Dust Transport over the Atlantic during the Last Two Decades (1979-1997). Geophysical Research Letters, 29, 17-20. https://doi.org/10.1029/2001GL013767

[9] Cakmur, R.V., Muller, R.L. and Tegen, I. (2001) A Comparison of Seasonal and Interannual Variability of Soil Dust Aerosols over the Atlantic Ocean as Inferred by the TOMS AI and AVHRR AOT Retrievals. Journal of Geophysical Research: Atmospheres, 106, 18287-18303. https://doi.org/10.1029/2000JD900525

[10] Aissani, O., Mokhnache, A. and Labbi, A. (2008) Etude des fluctuations du rayonnement solaire à partir de la modélisation et la télédétection passive. Revue des Energies Renouvelables, CICME'08, 19-26.

[11] Chen, M., Zhuang, Q.L. and He, Y.J. (2014) An Efficient Method of Estimating Downward Solar Radiation Based on the MODIS Observations for the Use of Land Surface Modeling. Remote Sensing, 6, 7136-7157. 
https://doi.org/10.3390/rs6087136

[12] Pinker, R.T., Niu, X.L. and Ma, Y.T. (2014) Solar Heating of the Arctic Ocean in the Context of Ice-Albedo Feedback. Journal of Geophysical Research: Oceans, 119, 8395-8409. https://doi.org/10.1002/2014JC010232

[13] Akana, N.L. and Njomo, D. (2010) Profiles of Cloud Fraction and Cloud Water Contents Deduced from Ground-Based Solar Radiation Measurements. Asia-Pacific Journal of Atmospheric Sciences, 46, 483-496. https://doi.org/10.1007/s13143-010-0029-9

[14] AFGL-TR-86-0110 (1986) Atmospheric Constituent Profiles (0-120km). Environmental Research Papers No. 954 ADA175173.

[15] Penner, S.S. (1960) Quantitative Molecular Spectroscopy and Gas Emissivities. Physics Today, 13, 38. https://doi.org/10.1063/1.3057073

[16] Rothman, L.S., Goldman, A., Gillis, J.R., Gamache, R.R., Pickett, H.M., Poynter, R.L., Husson, N. and Chedin, A. (1983) AFGL Trace Gas Compilation: 1982 Version. Applied Optics, 22, 1616-1627. https://doi.org/10.1364/AO.22.001616

[17] Rehle, D., Leleux, D., Erdelyi, M., Tittel, F., Fraser, M. and Friedfeld, S. (2001) Ambient Formaldehyde Detection with a Laser Spectrometer Based on Difference Frequency Generation in PPLN. Applied Physics B, 72, 947-952. https://doi.org/10.1007/s003400100549

[18] McCartney, E.J. (1976) Optics of the Atmosphere, Scattering by Molecules and Particles. Wiley, New York, 176-215.

[19] Oyola, M.I., Campbell, J.R., Xian, P., Bucholtz, A., Ferrare, R.A., Burton, S.P., Kalashnikova, O., Ruston, B.C. and Lolli, S. (2019) Quantifying the Direct Radiative Effect of Absorbing Aerosols for Numerical Weather Prediction: A Case Study. Atmospheric Chemistry and Physics, 19, 205-218.

https://doi.org/10.5194/acp-19-205-2019

[20] Young, A.T. (1981) Rayleigh Scattering. Applied Optics, 20, 533-535. https://doi.org/10.1364/AO.20.000533

[21] Peck, E.R. and Reeder, K. (1972) Dispersion of Air. Journal of the Optical Society of America, 62, 958-962. https://doi.org/10.1364/JOSA.62.000958

[22] Gueymard, C.A. (2001) Parameterized Transmittance Model for Direct Beam and Circumsolar Spectral Irradiance. Solar Energy, 71, 325-346. https://doi.org/10.1016/S0038-092X(01)00054-8

[23] Gueymard, C.A. (2004) The Sun's Total and Spectral Irradiance for Solar Energy Applications and Solar Radiation Models. Solar Energy, 76, 423-453. https://doi.org/10.1016/j.solener.2003.08.039

[24] Chen, M. and Zhuang, Q. (2004) Evaluating Aerosol Direct Radiative Effects on Global Terrestrial Ecosystem Carbon Dynamics from 2003 to 2010. Remote Sensing, 6, 7136-7157. https://doi.org/10.3390/rs6087136

[25] Eddounia, F. (2010) Cirrus Microphysics at the Global Scale: Correlation with Atmospheric Properties and Better Representation in Climate Models. Doctoral Thesis, Paris Institute of Technology, Paris.

[26] Chou, M.D. and Saurez, M.J. (1999) A Solar Radiation Parametrisation (CLIRADSW) for Atmospheric Studies. NASA/TM-1999-104606, Volume 15.

[27] Terasova, T.A. and Cavalcanty, I.F.A. (2000) Annual Cycle of Cloud Radiative Forcing over South America Simulations with CPTEC/COLA AGCM and SRB Data. Proceedings of 6 th International Conference on Southern Hemisphere Meteorology and Oceanography, AMS, Boston, 256-257. 
[28] Fana, Y. and Stamnes, K. (2017) Atmospheric Correction over Coastal Waters Using Multilayer Neural Networks. Remote Sensing of Environment, 199, 218-240. https://doi.org/10.1016/j.rse.2017.07.016

[29] King, M.D. and Harshvardhan (1986) Comparative Accuracy of Selected Multiple Scattering Approximations. Journal of the Atmospheric Sciences, 43, 784-801. https://doi.org/10.1175/1520-0469(1986)043<0784:CAOSMS>2.0.CO;2

[30] Akana, N.L. and Njomo, D. (2009) Spatial and Temporal Distributions of Downwelling Solar Radiation in Cameroon as Derived Using a Parameterized Solar Radiative Transfer Model in a Molecular Atmosphere. The JP Journal of Heat and Mass Transfer, 3, 73-93.

[31] LRE (1984) Mesures du rayonnement solaire au Cameroun. MESRES, 4, 122.

[32] LRE (1984) Mesures du rayonnement solaire au Cameroun. MESRES, 5, 122. 